\title{
EL PAPEL DE LA UNIVERSIDAD EN LA FORMACIÓN CÍVICA DE LOS ESTUDIANTES DE NIVEL LICENCIATURA, A TRAVÉS DE UN ESTUDIO DE CASO: UANL, ITESM Y UDEM (2016-2019)
}

\author{
Mariana Elizabeth Sánchez Tamez \\ Alma Rosa Saldierna Salas
}

\section{Resumen}

La universidad ha sido considerada un lugar para la generación de conocimiento y profesionistas. La forma de vida de los alumnos e incluso sus acciones, futuras está en gran manera influenciadas por lo recibido a través de la educación que ellos reciben en estas instituciones. En esta investigación se estudia el caso de la educación particular y pública en México, y se determinan algunos de los rasgos que la educación ha tenido dentro del ámbito nacional en la formación de ciudadanos e impartición de educación cívica. El estudio ser hace a través de un abordaje cualitativo en dónde se realiza una comparativa entre ambos tipos de instituciones y el papel de la universidad alrededor de la educación cívica y como esta ha afectado la recepción de los jóvenes ante la participación política y su aprovechamiento para su crecimiento como ciudadanos, es posible analizarlo a partir de tres diferentes universidades: Instituto Tecnológico y Estudios Superiores de Monterrey, campus Monterrey (ITESM), Universidad Autónoma de Nuevo León y Universidad de Monterrey (UDEM).

Palabras clave: Formación cívica, estudiantes, educación pública y privada 